\title{
Acoustic resolution photoacoustic Doppler flowmetry using a transducer array: optimising processing for velocity contrast
}

T. M. Bücking, P. J. van den Berg, S. Balabani, W. Steenbergen, P. C. Beard, et al. 


\title{
Acoustic resolution photoacoustic Doppler flowmetry using a transducer array: optimising processing for velocity contrast
}

\author{
T. M. Bücking ${ }^{a}$, P. J. van den Berg ${ }^{b}$, S. Balabani ${ }^{\text {, }}$, W. Steenbergen ${ }^{b}$, P. C. Beard ${ }^{a}$, J. Brunker ${ }^{a}$ \\ ${ }^{a}$ Department of Medical Physics and Biomedical Engineering, University College London, Gower \\ Street, London WC1E 6BT, UK; \\ ${ }^{\mathrm{b}}$ Biomedical Photonic Imaging, MIRA Institute for Biomedical Technology and Technical \\ Medicine, University of Twente, PO Box 217, 7500 AE Enschede, The Netherlands; \\ ${ }^{\mathrm{c}}$ Department of Mechanical Engineering, University College London, Gower Street, London \\ WC1E 6BT, UK
}

\begin{abstract}
This work demonstrates the first measurements of blood flow velocity using photoacoustic flowmetry (PAF) employing a transducer array. The measurements were made in a flow phantom consisting of a tube $(580 \mu \mathrm{m}$ inner diameter $)$ containing blood flowing steadily at physiological speeds ranging from $3 \mathrm{~mm} / \mathrm{s}$ to $25 \mathrm{~mm} / \mathrm{s}$. Velocity measurements were based on the generation of two successive photoacoustic (PA) signals using two laser pulses with a wavelength of $1064 \mathrm{~nm}$; the PA signals were detected using a 64-element transducer array with a $-6 \mathrm{~dB}$ detection bandwidth of 11-17 $\mathrm{MHz}$. We developed a processing pipeline to optimise a cross-correlation based velocity measurement method comprising the following processing steps: image reconstruction, filtering, displacement detection, and masking. We found no difference in flow detection accuracy when choosing different image reconstruction algorithms (time reversal, Fourier transformation, and delay-and-sum). High-pass filtering and wallfiltering were however found to be essential pre-processing steps in order to recover the correct displacement information. We masked the calculated velocity map based on the amplitude of the cross-correlation function in order to define the region of interest corresponding to highest signal amplitude. These developments enabled blood flow measurements using a transducer array, bringing PAF one step closer to clinical applicability.
\end{abstract}

Keywords: Flowmetry, blood flow, photoacoustic, optoacoustic, Doppler, cross-correlation, transducer array, image processing, masking

\section{INTRODUCTION}

Imaging blood flow velocity in deep tissue (on the order of centimetres) can provide insight into a multitude of pathologies affecting the vasculature. Quantifying flow in the microvasculature is of particular benefit, for example in monitoring the perfusion of tumours, which could be useful to predict and measure the efficacy of drugs. Deep tissue blood flow imaging is typically done using pulsed Doppler ultrasound (PD-US), but this is restricted to imaging larger vessels unless contrast agents are used. Microvessels are difficult to image using ultrasound without exogenous contrast since they exhibit low echogenicity; in addition it is challenging to detect the slow flow velocities therein as they are comparable to the background motion of the tissue.

Photoacoustic imaging (PAI) is a promising candidate that has the potential to overcome the limitations of PD-US, because its contrast is based on optical absorption, offering a much better differentiation of microvessels from the surrounding tissue. To image flow using the photoacoustic (PA) effect, two successive laser pulses are used to generate two PA signals. If optical absorbers (red blood cells, RBCs) have moved towards the detector in the meantime, the time of arrival of the second PA signal will be earlier. By cross-correlating the two PA signals in order to estimate the timeshift between them, the velocity of RBCs can be calculated assuming the sound speed and direction of flow relative to

Photons Plus Ultrasound: Imaging and Sensing 2017, edited by Alexander A. Oraevsky, Lihong V. Wang, Proc. of SPIE Vol. 10064, 100642M - (c) 2017 SPIE · CCC code: 1605-7422/17/\$18 · doi: 10.1117/12.2252939 
the transducer are known. This principle, known as acoustic resolution photoacoustic flowmetry (AR-PAF), has been demonstrated using single element transducers ${ }^{1}$, with recent results showing the feasibility of detecting the flow of whole human blood using a high frequency $(30 \mathrm{MHz})$ transducer element ${ }^{2}$.

Previous work has demonstrated the implementation of AR-PAF using a transducer array ${ }^{3}$, which is essential if the technique is to be adopted into a clinical setting. A clinical transducer array was used (centre frequency of $7.5 \mathrm{MHz}$ ), but measurements were performed using large polystyrene spheres in a low density suspension (63 $\mu \mathrm{m}$ at $4 \mathrm{vol} \%)$, which facilitates the measurements but is not representative of whole blood ${ }^{4}$.

Here, we demonstrate the feasibility of measuring blood flow using a clinical transducer array (centre frequency of 15 $\mathrm{MHz}$ ). These measurements are susceptible to noise and outliers, so we advanced the current PAF processing paradigm to make this imaging modality more robust. We looked at different image reconstruction methods, image filtering techniques and strategies in masking the data to remove signal originating from outside the region of interest.

\section{IMAGE ACQUISITION}

In order to mimic microscale blood flow, polyethylene tubing $(0.58 \mathrm{~mm}$ inner diameter $)$ was immersed vertically in a water bath. Two experiments were performed: a preliminary study using a suspension of polystyrene spheres in water $(10 \mu \mathrm{m}$ diameter, $0.5 \mathrm{vol} \%)$ and a second study using healthy human donor blood with $0.5 \quad \mathrm{M}$ Ethylenediaminetetraacetic acid (EDTA) added to prevent coagulation.

The tube was illuminated with a Q-switched Nd:YAG Litron Nano L PIV laser; two successive laser pulses were generated with a pulse separation of $\mathrm{T}=1 \mathrm{~ms}$. For the preliminary study using polystyrene spheres, the laser was frequency doubled with a harmonic crystal to provide excitation at $532 \mathrm{~nm}$ to improve the light absorption of the phantom. The measurements of whole blood were performed at $1064 \mathrm{~nm}$, as the lower absorption coefficient enabled a more homogeneous illumination of the tube. This step was necessary, because of the much higher concentration of RBCs in blood (about $45 \mathrm{vol} \%$ ) compared to the polystyrene phantom. The beam diameter at the tube was approximately $5 \mathrm{~mm}$ in diameter to simulate diffuse illumination.

The PA signal was detected with a 64 element ultrasound probe (ESAOTE SL3116), which has a pitch of $100 \mu \mathrm{m}$ and a bandwidth ranging from 11 to $17 \mathrm{MHz}$ at the $-6 \mathrm{~dB}$ points. The sampling rate was $50 \mathrm{MHz}$. The probe was positioned at an angle of $\theta=60^{\circ}$ to the tube, such that a cross-section of the tube was imaged (see fig. 1).

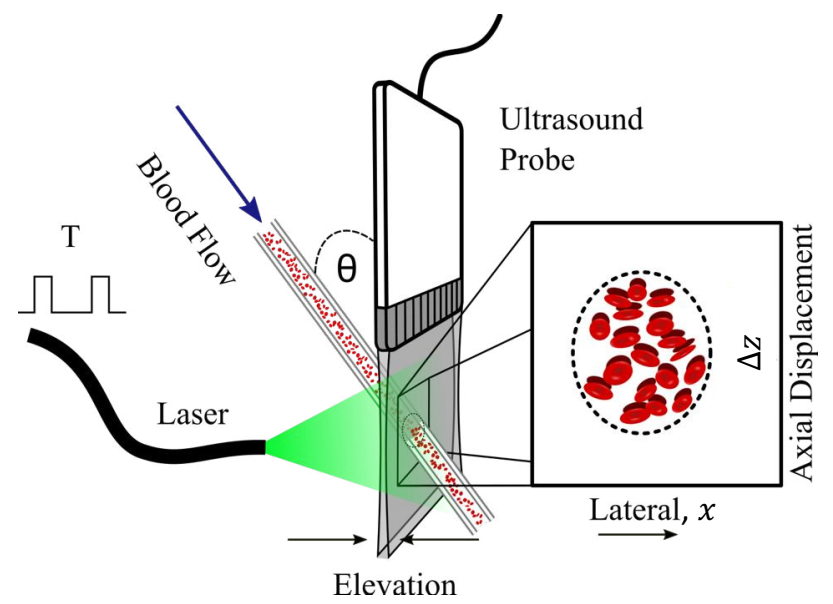

Figure 1. Illustration of the experimental setup. A polyethylene tube with $580 \mu \mathrm{m}$ diameter is diffusely illuminated with a Q-Switched Nd:YAG laser with two successive laser pulses. The laser triggers ultrasound acquisition of a 64-element ultrasound probe, detecting the PA signal of the absorbers in the tube (RBCs or polystyrene spheres). The probe images a cross-section of the tube, which means that absorbers would be displaced a distance $\Delta \mathrm{z}$ downwards between detected images due to the finite flow speed. $\theta=60^{\circ}$.

The flow speed was controlled using a syringe pump (Cole-Parmer WZ-74900-15), providing flow speeds in the range of $3 \mathrm{~mm} / \mathrm{s}$ to $25 \mathrm{~mm} / \mathrm{s}$. For each speed setting 200 image pairs were acquired, after which the syringe was flipped and 
high pressure was applied for a short amount of time, to prevent sedimentation inside the syringe and the tube. After flushing and setting the next flow speed, the flow was allowed to stabilise for one minute before acquiring the next data set.

There are a number of different methods to reconstruct a photoacoustic image from the raw RF data, which yield slightly different images. To ensure that the choice of reconstruction algorithm does not affect the accuracy of flow speed estimation, we investigated three different methods in a preliminary study using polystyrene spheres: Time reversal (TR $)^{5,6}$, Fourier transformation (FT) ${ }^{7}$ and Delay-and-Sum (DS). In subsequent experiments with whole blood, we employed the Fourier transformation reconstruction. The reconstructed pair of images of the $k$ th laser pulse pair will be denoted as $\boldsymbol{p}_{1}^{k}(x, z)$ and $\boldsymbol{p}_{2}^{k}(x, z)$, where $x$ and $z$ are the pixel indices in the lateral and axial direction respectively.

\section{IMAGE PROCESSING}

In the following, the processing steps that were carried out to calculate the flow velocities from the acquired PA image pairs will be described. The bold typeface denotes a vector (if one-dimensional), or an array (if multidimensional).

\subsection{Filtering}

After reconstruction, the images were filtered. Two filtering steps were employed: high-pass filtering and wallfiltering.

A zero-phase high-pass filter with a passband frequency of $5 \mathrm{MHz}$ was applied in the axial direction of the images to reduce noise. The $5 \mathrm{MHz}$ passband frequency is outside of the bandwidth of the detector. Moreover, frequency content below $5 \mathrm{MHz}$ is dominated by the structure of the tube. It is important to supress signal from the stationary structures in the images in order to enable unbiased motion detection.

The second filtering step (wallfiltering) involves subtracting the mean image:

$$
\mathrm{WF}\left(\boldsymbol{p}_{i}^{k}(x, z)\right):=\boldsymbol{p}_{i}^{k}(x, z)-\frac{1}{N} \sum_{k}^{N} \boldsymbol{p}_{i}^{k}(x, z),
$$

Where WF is the wallfilter functional and $N$ is the number of image pairs acquired with $i=\{1,2\}$. This step removes signal which remains constant between successive images and hence does not carry any flow information.

\subsection{Quantifying Flow}

After filtering, a 2D map of displacement is obtained by cross-correlating corresponding image segments ("interrogation windows") from successive images. We selected line segments which are 16 pixels long in the axial direction and one pixel wide in the lateral direction. This way, we only estimated the flow velocity component in the axial direction, as is also done in Doppler ultrasound imaging. Denoting these segments as interrogation windows, $\boldsymbol{w}_{i}(z)$, the normalised discrete cross-correlation is defined as

$$
\boldsymbol{R}_{k}^{r}(m):=\left\{\begin{array}{cc}
\frac{1}{L-|m|} \sum_{z=0}^{L-m-1} \boldsymbol{w}_{1}^{r}(z+m) \boldsymbol{w}_{2}^{r *}(z) & m \geq 0 \\
\boldsymbol{R}_{k}^{r *}(-m) & m<0
\end{array}\right.
$$

where $L$ is the length of the segments (16 pixels), the superscript $\boldsymbol{r}$ identifies the location of the centre of the window with respect to $\boldsymbol{p}(x, z)$, and $*$ denotes the complex conjugate.

Assuming uniform displacement across the interrogation window, the displacement of absorbers at $\boldsymbol{r}$ is given by the location of the peak value of the average cross-correlation function, also called the ensemble correlation $R_{\text {Ens }}^{r}$ :

$$
R_{\mathrm{Ens}}^{r}:=\frac{1}{N} \sum_{k}^{N} R_{k}^{r},
$$

where $R$ is the spline interpolation of the discretised $\boldsymbol{R}$. The estimated mean displacement of absorbers in the image segment, $\Delta z$, can be found using

$$
\frac{\Delta z}{\delta}:=\arg \max \left(R_{\text {Ens }}^{r}\right),
$$

where $\delta$ is a constant that is used to convert the number of pixels to a distance, i.e. it represents the length of one pixel. We find $\Delta z$ for every possible $\boldsymbol{r}$, i.e. every point in $\boldsymbol{p}(x, z)$ - except the top and bottom 8 rows, corresponding to half the length of $\boldsymbol{w}$ - creating a displacement map $\Delta \boldsymbol{z}(x, z)$. Thus, a velocity map can be constructed using 


$$
\boldsymbol{v}(x, z)=\frac{\Delta z(x, z)}{\mathrm{T} \cos (\theta)}
$$

$\boldsymbol{v}(x, z)$ does not discriminate between regions of high or low signal amplitude; even segments containing only noise will result in velocity estimates in that region athough these estimates will be spurious. To remove these estimates, the ROI is masked as described in the following section.

\subsection{Masking}

Masking is employed to reduce the velocity map to the region of interest: the tube. We investigated two masking strategies, both based on the maximum value of the cross-correlation function, $\max (\boldsymbol{R}) \cdot \max (\boldsymbol{R})$ is a good indicator for the region of interest, because it corresponds well with the photoacoustic signal amplitudes, $\boldsymbol{p}$. However, $\boldsymbol{p}$ can take very low values locally due to low SNR. In those regions, a mask based directly on $\boldsymbol{p}$ would fail to identify a region of interest. Because the extent of $\boldsymbol{w}$ is large in comparison to those regions of low amplitude in $\boldsymbol{p}$ and the cross-correlation function takes positive values if both $\boldsymbol{w}_{\mathbf{1}}(z+m)$ and $\boldsymbol{w}_{\mathbf{2}}(z)$ are negative or positive, a mask based on max $(\boldsymbol{R})$ is more robust to low SNR. This can be utilised by the following two approaches.

The first approach is to directly take the amplitude from the ensemble correlation:

$$
M_{\mathrm{Ens}}:=R_{\mathrm{Ens}}^{r}(\Delta z / \delta) \text {. }
$$

This can be prone to outliers because it is based on the average of the cross-correlation functions: If there is one $\max \left(R_{k}^{r}\right)$ with a very large outlier for any $k, M_{\mathrm{Ens}}$ will be skewed towards that value.

The second method investigated is the use of the median of the maxima of the cross-correlation functions:

$$
M_{\text {Med }}:=\operatorname{median}_{k}\left(\max \left(R_{k}^{r}\right)\right),
$$

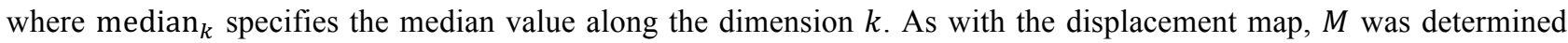
for every possible $\boldsymbol{r}$, thus constructing an amplitude map $\boldsymbol{M}(x, z)$.

Lastly, we employ a deconvolution step in constructing the final amplitude map. This is because every $M$, while corresponding to some location $\boldsymbol{r}$, was calculated using an image segment around $\boldsymbol{r}$, hence containing information about its immediate neighbourhood. By deconvolving $\boldsymbol{M}(x, z)$ with a top-hat function with a width of $L$, this effective blurring in the axial direction can be corrected for.

The amplitude map is thresholded at half its maximum value to serve as a mask for the velocity map (see fig. 2). At each flow speed setting, the velocity was estimated by calculating the median of the masked velocity map. The median was chosen due to its lower susceptibility to outliers. Error bars were based on the standard deviation of the flow estimates of the unmasked pixels in the map.
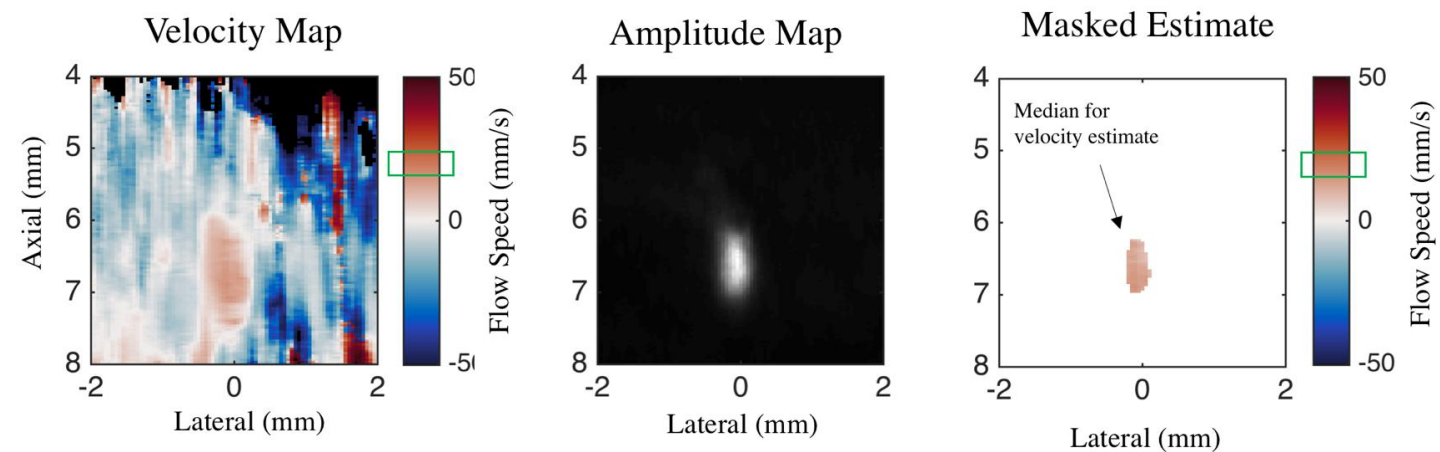

Figure 2: Illustration of masking photoacoustic velocity maps. The velocity map shows the correct flow region between a depth of 6 and $7 \mathrm{~mm}$ in the central region of the image (light red, highlighted with the green box in the colour bar). Velocity values are also calculated outside the region of interest where there is only nosie. Hence these are spurious random values. The amplitude map corresponds well with the cross-section of the tube and is thresholded to mask the velocity map. The median of the resulting masked estimate is taken as the final velocity estimate. 


\section{RESULTS AND DISCUSSION}

We investigated the effect of the different reconstruction methods (TR, FT, DS), the different filtering steps (high-pass filtering and wallfiltering), and the different masking methods $\left(\boldsymbol{M}_{\mathrm{Ens}}, \boldsymbol{M}_{\mathrm{Med}}\right)$, which were detailed in the previous section.

The preliminary study using polystyrene spheres was used to evaluate the effect of different reconstruction methods on the final velocity estimates. Figure 3 shows that we were successfully able to measure the flow of the spheres and that there was no significant difference in flow estimates between TR, FT, and DS image reconstruction. In these measurements both filtering steps were employed and masking was based on the deconvolved $\boldsymbol{M}_{\text {Med }}$. We chose FT as the reconstruction method for subsequent measurements as its computational implementation was the fastest of the three methods.

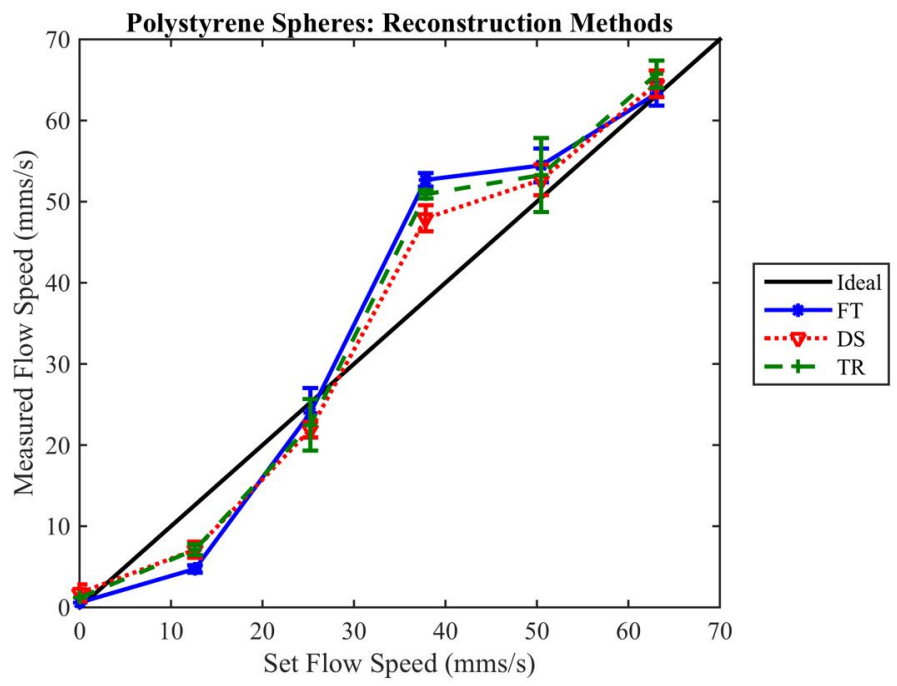

Figure 3: Photoacoustic flow measurements of polystyrene spheres in water $(10 \mu \mathrm{m}$ diameter, 0.5 vol\%) using a transducer array. Three different image reconstruction methods were used: Fourier Transformation (FT), Delay and Sum (DS), and Time Reversal (TR). As shown, the flow speeds were successfully recovered using all three algorithms, even though the reconstructions (not shown) were not identical in appearance.

Flow measurements using whole blood proved challenging and provided inaccurate estimates without any filtering (see blue data points plotted in fig. 4). However, using the wallfilter enabled accurate estimates of the flow speed. The highpass filter made the estimates more precise, because of the reduction in noise in $R_{\mathrm{Ens}}^{r}$.

Using $\boldsymbol{M}_{\mathrm{Ens}}(x, z)$ to calculate the masked velocity estimate proved unsuccessful, because outliers in $\boldsymbol{p}$, outside the region of interest, had such high amplitude in a few laser shots, that the mask became an inaccurate representation of the tube cross-section. Hence, the flow speed was estimated at the wrong location, yielding erroneous measurements (see, for example, the red data points plotted in fig. 4). $\boldsymbol{M}_{\mathrm{Med}}(x, z)$ on the other hand, was not influenced by the outliers, making it a robust enough masking technique which was necessary in this experiment. The green data points plotted in fig. 4 were calculated after both wallfiltering and high-pass filtering, and using $\boldsymbol{M}_{\mathrm{Med}}$ for masking, and they show consistently good agreement between the measured and known velocity values.

We also investigated the effect of deconvolving the amplitude map, which did not significantly change the results. However, deconvolution was required in order to correct for the axial distortion introduced by the cross-correlation computation and thus provide an accurate map of the flow region defined by the tube cross-section. 


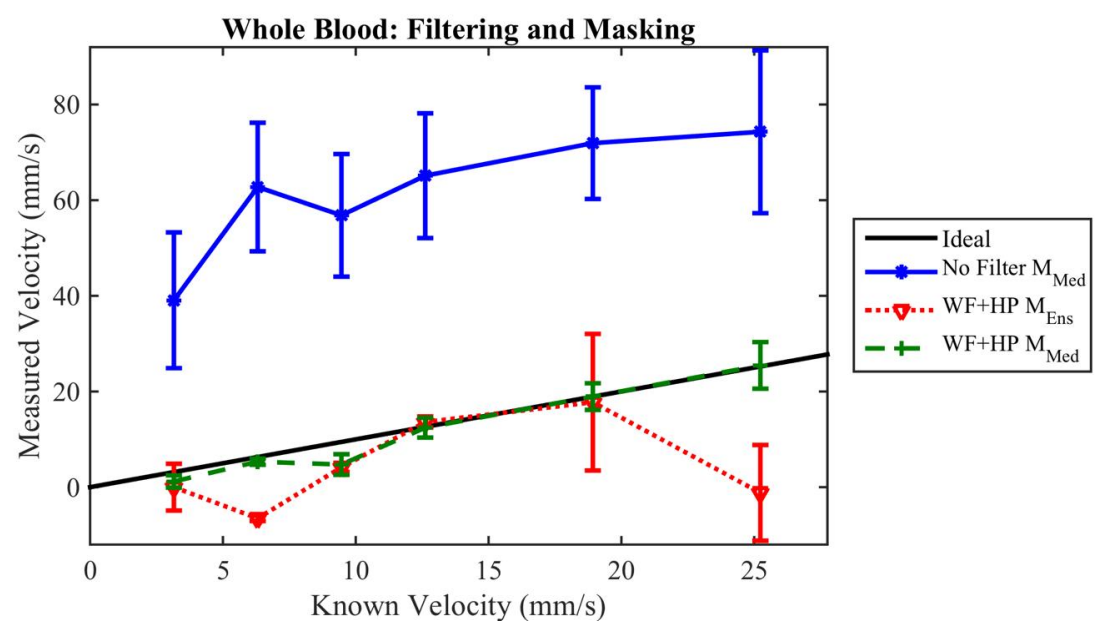

Figure 4: Photoacoustic flow measurements of human blood at $45 \%$ hematocrit and physiological flow speeds. The flow velocities were calculated from the reconstructed images using different masking approaches and with and without any filtering. Both wallfiltering and high-pass filtering were employed (WF+HP). Three different processing combinations are shown: using no filtering and using $\boldsymbol{M}_{\text {Med }}$ for masking (blue), employing both filtering steps and using $\boldsymbol{M}_{\text {Ens }}$ for masking (red), and employing both filtering steps and using $\boldsymbol{M}_{\text {Med }}$ for masking (green). Filtering is essential for accurate results. $\boldsymbol{M}_{\text {Med }}$ is less susceptible to outliers, which makes it the more robust masking strategy.

\section{CONCLUSIONS AND OUTLOOK}

We have successfully demonstrated that it is possible to measure the flow speed of human blood by detecting photoacoustic signals with a transducer array. This preliminary study, combining photoacoustic imaging (PAI) with acoustic resolution photoacoustic flowmetry (PAF), is a crucial step in bringing PAF closer to application in a clinical setting.

In order to enable these challenging blood velocity measurements, we have developed a processing pipeline which makes the technique robust enough to overcome the deleterious effects of a range of sources of noise and outliers. We have shown that the choice of image reconstruction algorithm has a negligible effect on the velocity estimates. On the other hand, filtering the reconstructed images has proved crucial for successful measurements: in particular, wallfiltering and high-pass filtering were shown to be necessary in order to supress background signal, which hinders motion detection. Lastly, we have developed a masking method to calculate the flow in the correct region of interest. This is not only relevant for quantification purposes. It also enables PAF information to be overlaid on to the PA structural image.

While we have demonstrated the feasibility of PAF for measuring the flow velocity of human blood, the source of velocity contrast remains an open question. Red blood cells (RBCs) are about $8 \mu \mathrm{m}$ in diameter, which is about an order of magnitude smaller than the wavelength of ultrasound in water, which at $20 \mathrm{MHz}$ (the upper frequency limit of signals detected in the present work) is about $75 \mu \mathrm{m}$. Furthermore, the RBC's concentration was about 45 vol\%, resulting in so densely packed cells that PAF cannot resolve the motion of individual RBCs. We conclude that we detected the motion of the larger RBC ensemble. There are two theories as to how this is possible. First, it might be the case that RBCs display heterogeneity on larger scales. This could, for example, be caused by RBC clustering, which happens at low blood flow velocities. Second, it is possible that some form of ultrasound speckle plays a role. While the extent to which speckle arises in PAI is a subject of contention ${ }^{8,9}$, its presence might facilitate the detection of motion regardless of RBC heterogeneity. 


\section{ACKNOWLEDGEMENTS}

This work is supported by the EPSRC-funded UCL Centre for Doctoral Training in Medical Imaging (EP/L016478/1), the Department of Health's NIHR-funded Biomedical Research Centre at Moorfields Eye Hospitals, and by the European Community's Seventh Framework Programme (FP7/2007-2013) under grant agreement nº 318067.

The authors would like to thank Litron for the use of their laser.

\section{BIBLIOGRAPHY}

[1] Brunker, J., and Beard, P., "Pulsed photoacoustic Doppler flowmetry using time-domain cross-correlation: accuracy, resolution and scalability," Journal of the Acoustical Society of America 132(3), 1780-1791 (2012).

[2] Brunker, J., and Beard, P., "Velocity measurements in whole blood using acoustic resolution photoacoustic Doppler," Biomedical Optics Express 7(7), 2789-2806 (2016).

[3] van den Berg, P.J., Daoudi, K., and Steenbergen, W., "Pulsed photoacoustic flow imaging with a handheld system.," Journal of Biomedical Optics 21(2), 26004 (2016).

[4] Brunker, J., and Beard, P., "Acoustic resolution photoacoustic Doppler flowmetry: practical considerations for obtaining accurate measurements of blood flow," in Proc. SPIE 8943, A. A. Oraevsky and L. V. Wang, Eds., 89431K-1-89431K-7 (2014).

[5] Xu, Y., and Wang, L. V., "Application of time reversal to thermoacoustic tomography," Proc. SPIE 5320, $257-$ 263 (2004).

[6] Treeby, B.E., Zhang, E.Z., and Cox, B.T., "Photoacoustic tomography in absorbing acoustic media using time reversal," Inverse Problems 26(11), 115003 (2010).

[7] Koestli, K.P., Frenz, M., Bebie, H., Weber, H.P., Köstli, K.P., Frenz, M., Bebie, H., and Weber, H.P., “Temporal backward projection of optoacoustic pressure transients using fourier transform methods," Physics in Medicine and Biology 46(7), 1863-72 (2001).

[8] Guo, Z., Li, L., and Wang, L. V., “On the speckle-free nature of photoacoustic tomography,” Medical Physics 36(9), 4084 (2009).

[9] Hysi, E., Dopsa, D., and Kolios, M.C., "Photoacoustic tissue characterization using envelope statistics and ultrasonic spectral parameters,” in Proc. SPIE 8943, 89432E-1-9 (2014). 